\title{
The Data Processing and Calibration of the AIRSAR PacRim II Mission
}

\author{
Anhua Chu, Wayne Tung, and Ellen O'Leary \\ Jet Propulsion Laboratory, \\ California Institute of technology, \\ Pasadena, CA 91109 USA \\ Anhua.chu@jpl.nasa.gov
}

\begin{abstract}
In this paper, we present the results of raw echo data analysis with data error recovery, data processing, and the calibration results of the Jet Propulsion Laboratory's airborne synthetic aperture radar (AIRSAR) for the Pacific Rim II mission. We also present the key elements of the calibration techniques for both polarimetric SAR (POLSAR) and cross-track interferometric SAR (TOPSAR) processed with the AIRSAR Integrated Processor.
\end{abstract}

\section{INTRODUCTION}

As part of NASA's Earth Science enterprise, AIRSAR data are currently collected for NASA-funded investigators in the United States as well as sponsors from international organizations. The Pacific Rim II mission took place in July through October 2000 . Over 60 hours of AIRSAR data were collected during the mission. The NASA/JPL AIRSAR system is a three-frequency airborne SAR system that was developed as a general test-bed for various advanced SAR techniques.

The NASA/JPL AIRSAR Integrated Processor (AIP) is a multi-frequency polarimetric and interferometric SAR processor designed to produce data useful for better understanding of scattering from different type of Earth terrain. The AIP is used to automatically generate co-registered multifrequency ( C-, L-, and P-band ) images from both polarimetric and interferometric data collection modes. The Digital Elevation Map ( DEM ) can be generated using the C-band or Lband interferometric data, then all other data channels are projected onto the ground using that DEM.

\section{DATA PROCESSING}

The AIRSAR data processing system consists of the survey image processor and the precision image processor. The survey data which show entire flight lines processed to a low resolution uncalibrated browse product were completed and posted on the web site ( http://airsar.jpl.nasa.gov ) by the time the instrument was returned to JPL in early November. The calibration of the standard modes was finalized in December and operational data processing was then started.

The AIRSAR precision data processing system is a window-driven system that consists of the following subsystems

- An internet Web-based product request Oracle database subsystem,

- An intranet product request queue management,

- Job setup module,

- Job submission module,

- A Sony high-speed tape downloading subsystem,

- The AIP,

- Quality assurance procedures, and

- CDROM maker.

After job completion, the queue is directly updated and investigators can browse the image gif files on their Web.

The first step of performing the calibration procedure is to verify and recover the raw echo data, auxiliary data and motion data. In the job setup module, the raw echo data is scanned and fixed line by line such as the anomalies range samples extraction and range samples shifting, and Radio Frequency Interference (RFI) filtering. For the PacRim II data, the rate of contaminated pulses was less than $0.1 \%$ and most of $80 \mathrm{MHz}$ L-band data had a 2-byte shift which is fixed during data processing. Most of the P-band data had RFI and some of L-band data as well. About $80 \%$ of the RFI can be removed with the current RFI filtering module. An improved version of the RFI filtering module is under evaluation and it will clean the RFI up to $90 \%$. The Ashtek Differential Global Position System (D-GPS) was incorporated into the AIP during the PacRim II mission along with the H764G Embedded GPS Receiver in an Inertial Navigation System (INS) (EGI) and the stability of motion data was increased over previous years.

\section{POLSAR CALIBRATION}


The second step is to perform the POLSAR calibration to generate backscatter in Stokes matrix form. External calibration devices (corner reflectors ) provide the absolute radiometric calibration[1]. During this step, the phase path compensation of the transmitter, receiver, and all antennas is calibrated and the cross-talk is also removed. Due to the path length differences among the four polarization combinations ( $\mathrm{HH}, \mathrm{HV}, \mathrm{VH}$, and $\mathrm{VV}$ ) in the radar hardware, a model has been developed [2] to identify the receiver path phase $(\Phi r)$, transmitter path phase $(\Phi t)$ and antenna path phase (Фa). Those three path phases measurements can be obtained from the SAR data with co-polarization and cross-polarization phases and the injected caltone phase( $\Phi$ cal). We have implemented cross-talk contribution by using eight adjacent pixels in each range line to get one estimate of the cross-talk parameters then averaging over the estimates of all the eight pixel blocks for each range line. What remains is to utilize the backscatter measurements from corner reflectors to correct the residual amplitude offsets in the various polarization channels and to correct for the absolute gain and co-polarized component phase of the radar system. In calculating the correlator gain, we use the theoretical expression for triangular trihedral corner reflector cross-section found in Ruck et al[4]

$\sigma=\frac{4 \pi}{\lambda^{2}} l^{4}\{\cos \theta+\sin \theta(\sin \phi+\cos \phi)-$

$\left.[\cos \theta+\sin \theta(\sin \phi+\cos \phi)]^{-1}\right\}^{2}$

Where: $l$ is the length of corner reflector sides,

$\lambda$ is the radar wavelength,

$\theta$ is radar wave incident angle, and $\phi$ is corner reflector azimuth angle.

For PacRim II, the POLSAR image absolute calibration for all 3 bands is within $3 \mathrm{~dB}$ and relatively calibrated between frequencies of 1.5 $\mathrm{dB}$. The plot shown below is the Rosamond's corner reflectors for the ratio of $\mathrm{VV} / \mathrm{HH}$ gain balance, the phase difference between VV and $\mathrm{HH}$ in degrees, and the absolute gain $(\mathrm{HH})$ difference between the measurement and theory in $\mathrm{dB}$ for the PacRim II $40 \mathrm{MHz}$ C-band POLSAR.

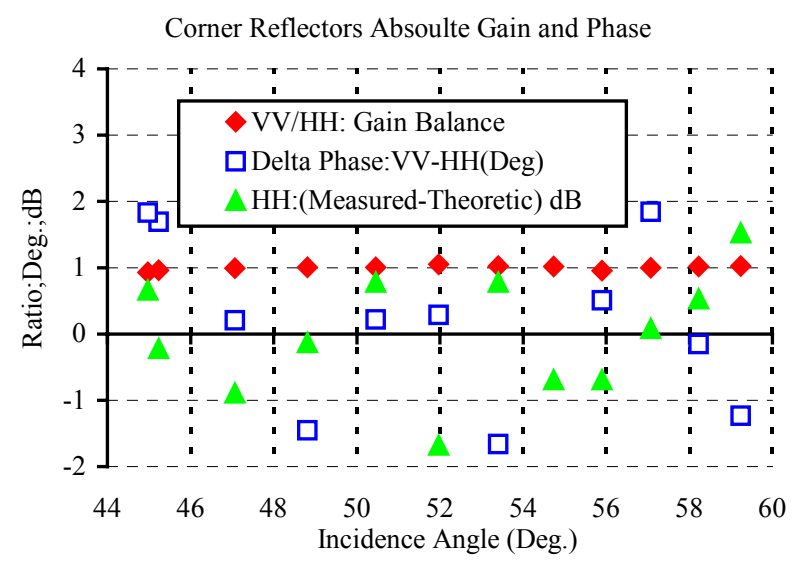

\section{TOPSAR CALIBRATION}

The third step is to calibrate the TOPSAR data for each pair of interferometric channels to generate the digital elevation models (DEMs). In our calibration of the interferometric SAR data, we determine the following parameters: time delay, the physical baseline length, baseline roll and yaw angles, and the differential phase. In practice, the time delay is divided into determining the differential time delay which between pairs of upper and lower data channels processed interferometrically and determining a common range delay. The baseline between the two antennas and the differential phase are calibrated using corner reflector ground truth position information. The C-band interferometry upper and lower antennas are separated by 2.5 meters with a roll angle of $50^{\circ}$ and a baseline yaw angle of $-0.5^{\circ}$. The L-band antennas are separated by 1.9 meters with a roll angle of $69^{\circ}$ and a yaw angle of $-2^{\circ}$. The calibration parameters are determined by fitting errors in corner reflector positions using the known [5] sensitivity of the target position to calibration parameter errors. Given the airplane position $\mathbf{P}$ vector, line of sight $\mathbf{n}$ and slant range $\rho$, the position to the corner reflectors $(\mathbf{T})$ can be obtained from $\mathbf{T}=\mathbf{P}+\rho \cdot \mathbf{n}$. The error in the interferometric measurement and interferometric phase can be written as

$$
\begin{aligned}
& \delta T=\delta P+\rho \cdot \delta n+\delta \rho \cdot n \\
& \delta \varphi=(-4 \pi / \lambda)[(\rho 1-\rho 2) \cdot \mathbf{n}]
\end{aligned}
$$


where $\rho 1$ and $\rho 2$ are slant range from upper and lower antennas, respectively.

From [5], the interferometric phase and DEM height can be expressed as below:

$$
\begin{aligned}
& \delta \phi=(4 \pi / \lambda) \cdot B \cdot \sin (\theta-\alpha) \\
& \mathrm{Z}(\mathrm{y})=\mathrm{h}-\rho \cos (\theta)
\end{aligned}
$$

For the interferometric phase, we estimate the baseline vector and differential phase by using the flat portion of the Rosamond lakebed with 663 meter site height in the WGS-84 projection. When a common flight track is used for processing both interferometric channels, the interferometric phase $\delta \phi$ can be rewritten as[6].

$$
\begin{aligned}
& \delta \phi=(-4 \pi / \lambda) \cdot\left(\hat{n}_{t}-\hat{n}_{r}\right) \cdot \bar{B}_{t}- \\
& \frac{4 \pi}{\lambda} \hat{n}_{r} \cdot \bar{b}+\Delta \phi+2 \pi m+\phi_{e}
\end{aligned}
$$

where $\lambda=$ wave length,

$$
\begin{aligned}
n_{t} & =\text { true unit look vector, } \\
n_{r} & =\text { reference unit look vector, } \\
B_{t} & =\text { true baseline vector, } \\
\mathrm{b} & =\text { baseline error vector, } \\
\Delta \phi & =\text { differential phase } \\
\mathrm{m} & =\text { absolute phase number, and } \\
\phi_{e} & =\text { phase due to earth curvature. }
\end{aligned}
$$

When the reference flat height is the same as the true height, the first term of (6) becomes zero. After the earth curvature corrected and the phase ambiguity number (m) are determined, the resulting phase $\phi c$ can be written as

$$
\phi_{c}=\delta \phi-\phi_{e}=-\frac{4 \pi}{\lambda} \hat{n}_{r} \cdot \bar{b}+\Delta \phi
$$

Using the TOPSAR data, we can determine the baseline error vector (b) and differential phase $(\Delta \phi)$ with a least square error technique.

For the PacRim II, the relative height accuracy of the TOPSAR data is within 2 to $5 \mathrm{~m}$ for C-band and 5 to $10 \mathrm{~m}$ for L-band .

The plot shown on this page at right side top is the locations of the Rosamond corner reflectors for C-band $40 \mathrm{MHz}$ TOPSAR data with heading 350 degrees and the mean of the location error is around 1.5 meters.

\section{Corner Reflectors Location Offset}

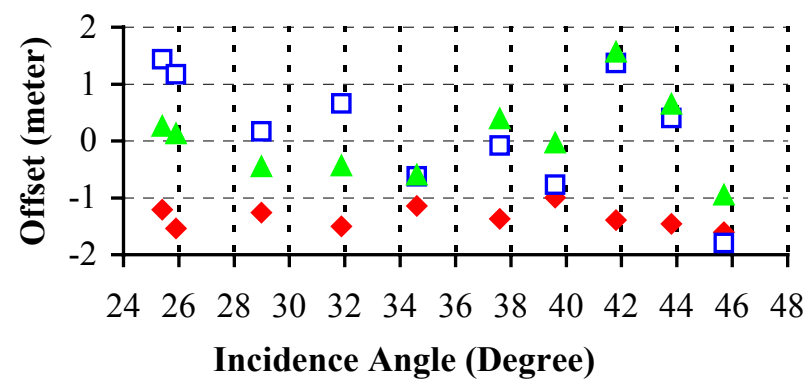

$\checkmark$ Along-track (mean:-1.4;sd:0.1) $\quad \square$ Cross-track (mean:0.2;sd:0.9)

$\Delta$ Height (mean:0.05;sd:0.5)

\section{CONCLUSIONS}

In this paper, we present the results of raw echo data analysis with error recovery and data processing, and the calibration results of standard AIRSAR modes. All AIP products are calibrated and corrected geometrically (TOPSAR mode), polarimetrically, and radiometrically. A number of precision image data sets will be presented to demonstrate the calibration for the PacRim II data.

\section{ACKNOWLEDGMENT}

The research described in this paper was carried out by the Jet Propulsion laboratory, California Institute Technology, under a contract with the National Aeronautics and Space Administration.

\section{REFERENCES}

[1]Van Zyl, J.Jakob. , Calibration of Polarimetric Radar Images Using Only Image Signatures and Trihedral Corner Reflectors Responses, IEEE Transactions on Geoscience and Remote Sensing, GE-28, pp.337-348, 1990.

[2]. Zebker,Howard,Lou, Yunling, Phase calibration of image radar polarimeter Stokes matrices, IEEE Trans. Geo. Remote Sensing, Vol. 28 pp.264-252 Mar. 1990.

[3] Van Zyl, J.J.et al., Image Radar polarimatric Signatures:Theory and Observations, Radio Science, 22 pp. 529-543, 1987.

[4] Ruck, G.T.,et al.,Radar Cross Section Handbook, vol. I, p.593, Plenum, New York, 1970.

[5] Madsen, Soren et al.,Topographic Mapping Using Radar Interferometry: Processing techniques, IEEE Trans, Geosci. Remote Sens., GRS-31 pp.246-256, 1993.

[6] Kim, Yunjin and Chu, Anhua "AIRSAR TOPSAR Calibration ", JPL Internal Memorandum, June, 1998 